\title{
THE HYDRAULIC ARCH*
}

\author{
By CHIA-SHUN YIH (University of Michigan)
}

Exact solutions for free surface flows are rare, especially if gravity is taken onto account. In this note an exact solution is given for a flow of which every streamline can be a free streamline.

As usual, $u$ and $v$ denote the velocity components in the directions of increasing $x$ and $y$, respectively, $x$ and $y$ being Cartesian coordinates, with $y$ increasing in the direction of the vertical. The gravitational acceleration, assumed constant in this note, will be denoted by $g$, and the density and the pressure will be denoted by $\rho$ and $p$, respectively.

Effects of viscosity will be neglected. For steady flows, the equations of motion are then

$$
\begin{aligned}
u u_{x}+v u_{y} & =-(1 / \rho) p_{x}, \\
u v_{x}+v v_{y} & =-(1 / \rho) p_{y}-g .
\end{aligned}
$$

The equation of continuity is, under the assumption of incompressibility,

$$
u_{x}+v_{y}=0 .
$$

The subscripts in the equations above indicate partial differentiation.

The simple solution

$$
u=U \text { (constant), } \quad v=-(g x / U)
$$

satisfies (3). Substituting (4) into (1) and (2), we obtain

$$
p_{x}=0=p_{y},
$$

whatever the value of $\rho$. Hence, for any density variation from streamline to streamline,

$$
p=\text { constant }
$$

throughout the whole field of flow, and in particular along any streamline.

The flow given by (4) is rotational, with vorticity equal to $-g / U$. The streamlines are given by

$$
\psi / U=y+\left(g / 2 U^{2}\right) x^{2},
$$

where $\psi$ is the stream function. They are parabolas with a vertical axis, obtainable from a single parabola by vertical displacement.

As a consequence of (6), the fluid above or below any streamline, or between any two streamlines, can be removed without affecting the rest of the flow. Furthermore, the density distribution, subjected to the sole restriction that $\rho$ must be constant along

* Received September 4, 1972. This work has been supported by the Office of Naval Research. 


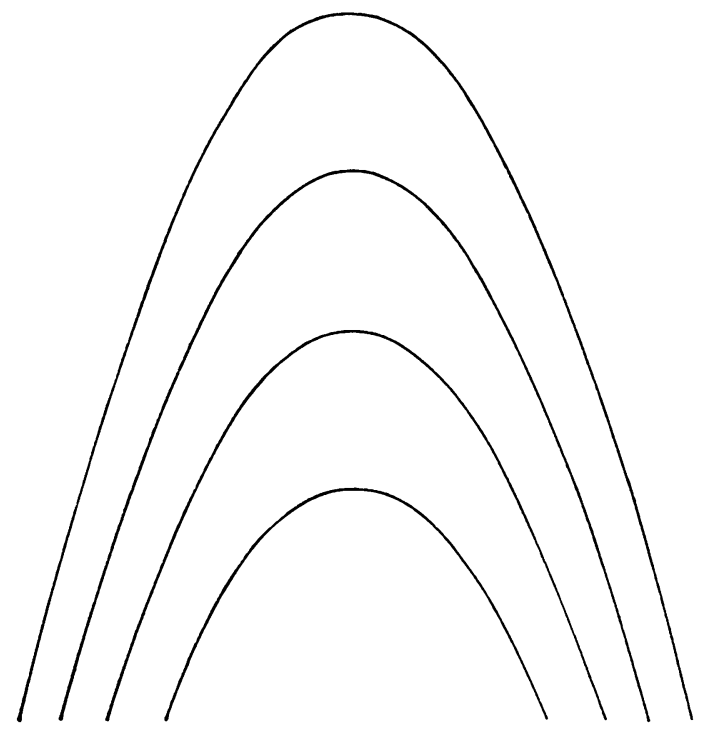

FIG. 1.

any streamline, may vary not only in any $x-y$ plane, but with $z$ (the third Cartesian coordinate) in any manner as well. Consequently, any portion of the fluid bounded by stream surfaces (in which streamlines are imbedded) can be removed without affecting the rest of the flow.

In particular, one can have a flow between two parabolas. This is a flow in a "hydraulic arch".

If the viscosity $\mu$ is constant, (4) still satisfies the Navier-Stokes equations, but the boundary conditions at the free surface (or free surfaces) are violated. However, if the Reynolds number (suitably defined) is large, the viscous effects will be concentrated in a thin layer (or in thin layers) at the free surface (or free surfaces).

Note also that if viscous effects are neglected the density along any streamline does not even need to be constant. If it is not constant the density will be unsteady, though the velocity field is steady. 